\author{
MILITARY TECHNICAL COLLEGE \\ CAIRO - EGYPT
}

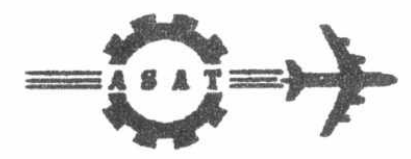

$7^{\text {th }}$ INTERNATIONAL CONF. ON AEROSPACE SCIENCES \& AVIATION TECHNOLOGY

\title{
Prediction of the Performance of a Backward-Curved Blade Centrifugal Pump
}

\author{
W.A. Wahba", H.M. Abdalla"* , M.A. El-Senbawi ${ }^{* * *}$
}

\begin{abstract}
Theoretical and experimental investigations have been made to predict the performance of a backward-curved blade centrifugal pump. A mathematical model based on steady twodimensional incompressible Navier-Stokes $(\mathrm{N}-\mathrm{S})$ equations has been developed. The numerical solution was made using the primitive variables with artificial compressibility. The predictorcorrector method proposed by MacCormack was employed. The use of this technique involved imaginary rows behind walls, and periodic boundaries at far upstream and downstream which adequately improved the convergence to the solution. Based on this model a computer code has been developed and used to predict the flow pattern inside the pump and to determine the pump characteristics. A test rig was used to measure the real pump characteristic at controlled flow rates. Comparison of calculated and measured pump characteristic showed good agreement. Examination of the flow pattern at different flow rates might be useful to interpret the many performance features of the pump.
\end{abstract}

\section{KEYWORDS}

Flow Pattern - Backward-Curved Blade - Centrifugal Pump - Computational Fluid Dynamics. Pump Performance - Experimental.

\section{INTRODUCTION}

The backward-curved blade centrifugal pump is commonly used for its quitter operation and more stable relationship between the capacity and the pumping head. The velocity of the liquid flow experiences a smoother change and a more uniform distribution in the impeller passage as compared to the case of radial blade. This gives rise to a reduction in the hydraulic losses in the impeller. The theoretical solution of the flow pattern inside a radial blade centrifugal pump is presented in reference [1]. The computational-fluid-dynamic scheme was based on primitive variables, artificial compressibility and MacCormack predictor-corrector technique. The

\footnotetext{
* Assistant, M.Sc., Department of Rockets, MTC, Cairo, Egypt.

** Assoc.Prof., Head of the Department of Rockets, MTC, Cairo, Egypt.

*.* Ph.D., Department of Rockets, MTC, Cairo, Egypt.
} 
scheme was then modified to cope with the more sophisticated problem of backward-curved blade impeller.

\section{MAIN GOVERNING EQUATIONS}

Consider the polar-coordinate system $(r, \theta)$, where $r$ denotes the radial direction and $\theta$ denotes the tangential direction. Consider the two-dimensional, incompressible N-S equations for a constant property flow without body forces or external heat addition. Using the concept of artificial compressiblity [2], the continuity equation, written in the system relative to a blade row, is:

$$
-\frac{\partial}{\partial t}(p)=\rho \delta\left[\frac{1}{r} \frac{\partial}{\partial r}\left(\mathrm{ru}_{\mathrm{r}}\right)+\frac{1}{\mathrm{r}} \frac{\partial}{\partial \theta}\left(\mathrm{u}_{\theta}\right)\right]
$$

where:

$u_{r}, u_{\theta} \quad$ are relative flow velocity components in $r$ and $\theta$ directions respectively.

$\rho \quad$ is the operating fluid density.

$\delta \quad$ is an artificial compressibility factor (constant).

After the above assumptions, the momentum conservation law for a rotating blade is the N-S equations written as [3]:

$$
\begin{gathered}
\frac{\partial}{\partial t}\left(u_{r}\right)+\frac{1}{r} \frac{\partial}{\partial r}\left(r u_{r}^{2}\right)+\frac{1}{r} \frac{\partial}{\partial \theta}\left(u_{r} u_{\theta}\right)-\frac{u_{\theta}^{2}}{r}-r \omega^{2}-2 \omega u_{\theta}=-\frac{1}{\rho} \frac{\partial p}{\partial r}+v\left[\nabla^{2} u_{r}-\frac{u_{r}}{r^{2}}-\frac{2}{r^{2}} \frac{\partial u_{\theta}}{\partial \theta}\right] \\
\frac{\partial}{\partial t}\left(u_{\theta}\right)+\frac{1}{r} \frac{\partial}{\partial r}\left(r_{r} u_{\theta}\right)+\frac{1}{r} \frac{\partial}{\partial \theta}\left(u_{\theta}^{2}\right)+\frac{u_{r} u_{\theta}}{r}+2 \omega u_{r}=-\frac{1}{\rho r} \frac{\partial p}{\partial \theta}+v\left[\nabla^{2} u_{\theta}-\frac{u_{\theta}}{r^{2}}+\frac{2}{r^{2}} \frac{\partial u_{r}}{\partial \theta}\right]
\end{gathered}
$$

where:

$\mathrm{p} \quad$ is static pressure.

$\omega \quad$ is the angular velocity of the rotating blade.

$v \quad$ is the kinematic viscosity.

$\nabla^{2}()=\frac{\partial^{2}}{\partial \mathrm{r}^{2}}()+\frac{1}{\mathrm{r}} \frac{\partial}{\partial \mathrm{r}}() \frac{1}{\mathrm{r}^{2}} \frac{\partial^{2}}{\partial \theta^{2}}() \quad$ (for polar coordinates)

The artificial compressibility factor $\delta$ can be obtained from[1]:

$$
\delta \geq\left(u_{r}^{2}+u_{\theta}^{2}\right)_{\max }
$$

There are two approaches to calculate incompressible N-S equations: implicit and explicit techniques. We will use the explicit one for which the computational procedures are relatively easy to implement. The most appropriate system of equations in differential form is the Reynolds averaged $\mathrm{N}-\mathrm{S}$ equations in a rotating polar-coordinate system given by Lakshminarayana [4].

$$
\frac{\partial \mathbf{Q}}{\partial t}+\frac{1}{r} \frac{\partial(r \mathbf{E})}{\partial \mathrm{r}}+\frac{1}{\mathrm{r}} \frac{\partial \mathbf{F}}{\partial \theta}=\frac{1}{\mathrm{r}} \mathbf{S}+\text { ViscousTerm }
$$

where: 
Q is the conservation variable.

$\mathbf{E}, \mathbf{F}$ are the flux vectors.

$S$ is the source term.

The viscous term will be discarded for its later use as central difference form in $\mathrm{r}$ and $\theta$ directions. Equation (5) becomes:

$$
\frac{\partial \mathbf{Q}}{\partial t}=-\frac{1}{r} \frac{\partial(\mathbf{r} \mathbf{E})}{\partial \mathrm{r}}-\frac{1}{\mathrm{r}} \frac{\partial(\mathbf{F})}{\partial \theta}+\frac{1}{\mathrm{r}} \mathbf{S}
$$

Using Equations $(1,2,3)$ yields

$$
Q=\left[\begin{array}{c}
P \\
u_{r} \\
u_{\theta}
\end{array}\right], \quad \mathbf{E}=\left[\begin{array}{c}
\rho \delta u_{r} \\
u_{r}^{2}+p / \rho \\
u_{r} u_{\theta}
\end{array}\right], \quad \mathbf{F}=\left[\begin{array}{c}
\rho \delta u_{\theta} \\
u_{r} u_{\theta} \\
u_{\theta}^{2}+p / \rho
\end{array}\right] \text {, and } \quad S=\left[\begin{array}{c}
0 \\
u_{\theta}^{2}+p / \rho+\omega^{2} r^{2}+2 \omega r u_{\theta} \\
-u_{r} u_{\theta}-2 \omega r u_{r}
\end{array}\right]
$$

where the elements $[p / \rho]$ and $[p / \rho]$ in the flux vector $\mathbf{E}$ and source term $S$ respectively are obtained from substituting $\left[-\frac{1}{\rho} \frac{\partial \mathrm{p}}{\partial \mathrm{r}}\right]$ in equation (2) with $\left[\frac{1}{\mathrm{r}} \frac{\mathrm{p}}{\rho}-\frac{1}{\mathrm{r}} \frac{\partial}{\partial \mathrm{r}}(\mathrm{r} \mathrm{p} / \rho)\right]$.

\section{NUMERICAL SOLUTION}

\subsection{Blade Profile And Computational Grid}

The present scheme calculates the flow through one blade passage. The blade profile is defined in the polar coordinate system by identifying the degree of a polynomial which best fits the profile of a real pump. Practically, this can be done by photoscanning a pump sector with two adjacent blades, using AutoCad package to interpret the scanned profile as a table, and then using GRAPHER package to transfer table data into a polynomial. In the studied case this procedure has lead to a $4^{\text {th }}$ degree polynomial The computational domain presented in a polar-coordinate system, is discretized into mesh points, Fig. 1. For this computational grid, the following definitions are applied: $\Delta \phi_{i}=\phi_{i+1}-\phi_{i}$ is the incremental angle in $\phi$ direction; $\alpha_{i, j}=r_{i+1} \Delta \phi_{i}$ is the arc length between points $A$ and $(i+1, j) ; \gamma_{i, j}=r_{i-1} \Delta \phi_{i-1}$ is the arc length between points $B$ and $(i-1, j) ; S_{i+1}=r_{i+1} \Delta \theta$ is the arc length between points $(i+1, j-1)$ and $(i+1, j)$; and $S_{i-1}=r_{i-1} \Delta \theta$ is the arc length between points $(i-1, j+1)$ and $(i-1, j)$.

The computational boundaries comprise the upstream inlet, the downstream exit, the pressure and suction side blade surfaces. The blade surfaces are extended along a surface of grid points in the upstream and downstream directions in a way similar to that described in ref.[1]. These form permeable periodic boundaries.

The properties at the points A and B can be computed in terms of their values at 3 neigbouring points applying quadratic interpolation [5].

$$
Q_{A}=\frac{\alpha_{i, j}}{2 S_{i+1}}\left[1+\frac{\alpha_{i, j}}{S_{i+1}}\right] Q_{i+1, j+1}+\left[1-\left(\frac{\alpha_{i, j}}{S_{i+1}}\right)^{2}\right] Q_{i+1, j}+\frac{\alpha_{i, j}}{2 S_{i+1}}\left[\frac{\alpha_{i, j}}{S_{i+1}}-1\right] Q_{i+1, j-1}
$$




$$
Q_{B}=\frac{\gamma_{i, j}}{2 S_{i-1}}\left[1+\frac{\gamma_{i, j}}{S_{i-1}}\right] Q_{i-1, j-1}+\left[1-\left(\frac{\gamma_{i, j}}{S_{i-1}}\right)^{2}\right] Q_{i-1, j}+\frac{\gamma_{i, j}}{2 S_{i-1}}\left[\frac{\gamma_{i, j}}{S_{i-1}}-1\right] Q_{i-1, j+1}
$$

\subsection{Finite Difference Scheme}

For calculation of $\mathbf{Q}$, a predictor step is carried out followed by a corrector step. The predictor and corrector equations are, respectively:

$$
\begin{aligned}
& \overline{\mathbf{Q}_{i, j}^{\mathrm{n}+1}}=\mathbf{Q}_{\mathrm{i}, \mathrm{j}}^{\mathrm{n}}-\frac{\Delta \mathrm{t}}{\Delta \mathrm{r}}\left[\mathbf{E}_{\mathrm{A}}^{\mathrm{n}}-\mathbf{E}_{\mathrm{i}, \mathrm{j}}^{\mathrm{n}}\right]-\frac{\Delta \mathrm{t}}{\mathrm{r}_{\mathrm{i}} \Delta \theta}\left[\mathbf{F}_{\mathrm{i}, \mathrm{j}+1}^{\mathrm{n}}-\mathbf{F}_{\mathrm{i}, \mathrm{j}}^{\mathrm{n}}\right]+\frac{\mathbf{S}_{\mathrm{i}, \mathrm{j}} \Delta \mathrm{t}}{\mathrm{r}_{\mathrm{i}}} \\
& \mathbf{Q}_{i, j}^{n+1}=\frac{1}{2}\left\{\mathbf{Q}_{i, j}^{n}+\overline{\mathbf{Q}_{i, j}^{n+1}}-\frac{\Delta t}{\Delta r}\left[\overline{\mathbf{E}_{i, j}^{n+1}}-\overline{\mathbf{E}_{\mathrm{B}}^{n+1}}\right]-\frac{\Delta t}{r_{i} \Delta \theta}\left[\overline{\mathbf{F}_{i, j}^{n+1}}-\overline{\mathbf{F}_{i, j-1}^{n+1}}\right]+\frac{\mathbf{S}_{i, j} \Delta t}{r_{i}}\right\}
\end{aligned}
$$

The parameters at the points $\mathrm{A}$ and $\mathrm{B}$ will be calculated using equations 8 and 9 respectively. The set of equations are solved following the same solution steps in [1]. The following considerations are taken into account:

- A smoothing term is added in order to suppress high frequency oscillations. This is accomplished by adding a fourth-order explicit dissipation term to the primitive variables in the two-directions of flow $(r, \theta)$ for interior points.

$$
-\varepsilon_{\mathrm{e}}\left[(\Delta \mathrm{r})^{4} \frac{\partial^{4}}{\partial \mathrm{r}^{4}}(\mathbf{Q})+(\Delta \theta)^{4} \frac{\partial^{4}}{\partial \theta^{4}}(\mathbf{Q})\right]
$$

where $\varepsilon_{\mathrm{e}}$ is the explicit smoothing coefficient.

The value of $\varepsilon_{e}$ should be less than approximately $1 / 16$ for stability [6].

- The stability condition is

$$
\Delta t=\left(\frac{2 F_{t}}{\frac{\left|u_{r}\right|}{\Delta r}+\frac{\left|u_{\theta}\right|}{r \Delta \theta}+4 v\left(\frac{1}{(\Delta r)^{2}}+\frac{1}{(r \Delta \theta)^{2}}\right)+\sqrt{\left(\frac{u_{r}}{\Delta r}+\frac{u_{\theta}}{r \Delta \theta}\right)^{2}+4 \delta\left(\frac{1}{(\Delta r)^{2}}+\frac{1}{(r \Delta \theta)^{2}}\right)}}\right)
$$

where $F_{t}$ is the time factor. It is found from experience that the time factor up to 0.9 can be used. In case of an unstable solution, the time factor is reduced by 0.1 .

Time steps are calculated based on the initial conditions and are not updated during the calculations. Thus, the time step varies as a function of grid spacing only. The iteration process is repeated until it converges.

- The computation is considered to be converged when the root mean square (RMS) of the residual in the velocity component $u_{r}$ drops below $10^{-5}$.

$$
R M S=\sqrt{\frac{\sum_{i=1}^{N i-1} \sum_{j=1}^{M}\left|u_{i_{i, j}+1}^{n+1}-u_{r_{i, j}}^{n}\right|^{2}}{(N i-1)(M)}}
$$


where:

$\mathrm{Ni}$ is the total number of grid lines from upstream to downstream extensions in $\mathrm{r}$ direction.

$\mathrm{M}$ is the number of grid lines from blade-to-blade in $\theta$ direction.

- The far upstream and downstream boundaries are located about half-chord, and one-chord respectively. On the upstream boundary the relative velocity components $u_{r}$ and $u_{\theta}$ are specified. On the downstream boundary only the static pressure $p$ is required. The other variables at both upstream and downstream boundaries are to be obtained by interpolation from the interior points.

- The wall points are considered as if they were interior points for the calculation of all the variables. This required to add a grid line before the pressure side and a grid line after the suction side. The parameters at these lines were obtained by quadratic interpolation from the values at the wall and two interior points. This is numerically exactly the same as applying the conservation equations to a point on the half spacing near the wall and then extrapolating from this point to the boundary [7]. To simulate blade row conditions, it is essential to impose zero radial and tangential velocities in case of $\mathrm{N}-\mathrm{S}$ equations. At the periodic boundary the variables were calculated as the interior points. In this case the periodicity condition could be used to obtain the variables which were located beyond the boundary. The results at corresponding points were then averaged after each time step.

- For the initial conditions, the data required for the solution are the major impeller dimensions, the blade profile, the flow rate, the fluid viscosity and density, and the impeller speed. The radial velocity is obtained from continuity. The tangential velocities in upstream and downstream are obtained from Euler's equation. Inside the blade passage, the initial tangential velocity is determined by assuming that the relative velocity is tangent to the blade. Assuming reasonable starting value for the downstream static pressure, Bernoulli constant at downstream is calculated. The initial values of the static pressure at all the grid points can be readily calculated.

\subsection{Computer Code}

The developed program is written in the $\mathrm{C}++$ language (version 3.1 for Windows $3 . \mathrm{x}$ ) with a total of about 800 statements. The execution file requires about 90 Kbytes of storage. Up to $52 \times 23$ grid points maximum, with a corresponding memory requirements of approximately 8 Mbytes, can be used. Time requirements per point per time step were about $4.7 \times 10^{-7}$ seconds when a computer of type PS-75MHz was used.

\section{EXPERIMENTAL WORK}

A test rig equipped with centrifugal pump and the appropriate measuring instruments has been used for investigating the pump characteristics. A photograph of the test rig is shown in Fig.2. The corresponding schematic diagram indicating the major components of the test rig, is shown in Fig. 3. The centrifugal pump under consideration is of the single stage, one shroud type in which a single impeller rotates in a volute casing. Fluid enters the impeller axially through the eye, spirals outward and discharges radially from the impeller circumference into 
the volute casing. The pump is driven by means of a DC motor of variable speed with a controller which allows the pump speed to be varied from zero to 3000 r.p.m.

\section{RESULTS AND DISCUSSION}

Based on the above scheme, a computer code was developed using the impeller with inner radius $0.0235 \mathrm{~m}$, outer radius $0.065 \mathrm{~m}$, number of blades is 6 and blade width is $0.012 \mathrm{~m}$. The water was used as the working fluid, with a flow rate ranging from 1 to $5 \mathrm{lit} / \mathrm{s}$ and a pump speed of $1500 \mathrm{rpm}$.

The convergence was predicted for flow rates $0.001,0.002,0.003,0.004$ and $0.005 \mathrm{~m}^{3} / \mathrm{s}$. Figure 4 shows the convergence histories for flow rate $0.004 \mathrm{~m}^{3} / \mathrm{s}$. Figure $4 \mathrm{a}$ shows the convergence of the RMS error in the radial velocity component. About 3100 iterations were required to obtain converged solution (RMS error $=10^{-5}$ ). The convergence of the outlet-toinlet flow rate ratio is shown in Fig. 4b. It is clear that the ratio is very close to one. The convergence of the radial velocity component near the leading edge is shown in Fig. 4c. Figure $4 \mathrm{~d}$ shows the convergence of the static pressure at the upstream boundary.

Figure 5 shows the difference between the theoretically determined static characteristics and the experimentally measured one. As seen from this graph, it is clear that the behavior of both graphs is almost similar, but the theoretical values of the pump head is greater than the measured values. This change in magnitude may be explained by the pump losses during the pump operation. In the theoretical head the only losses considered are the impeller losses due to viscous effect between the fluid and the impeller vanes. During experimental work, additional losses include shock and friction losses in the volute casing. At low flow rates separation and shock losses are dominant. The static pressure distribution on the pressure and suction sides of the impeller is shown in Fig. 6.

Also, distributions of relative velocities and pressure were calculated for the above flow rates. The results for a flow rate of $0.004 \mathrm{~m}^{3} / \mathrm{s}$ are shown in Fig. 7. Figure 7a shows the radial relative velocity inside blade passage. The more developed flow downstream can be explained by larger flow area. Figure $7 \mathrm{~b}$ shows the tangential relative velocity inside blade passage. It can be shown that the difference of the tangential relative velocity across the radial direction becomes more pronounced near the pressure side. The reverse flows are well indicated at this flow rate. Figure $7 \mathrm{c}$ shows the pressure contours inside blade passage. It is clear that for the same grid line, the pressure at the pressure side is higher than that at the suction side. The change is more important as one goes upstream.

The flow pattern at different flow rates is shown in Fig. 8 . It could be seen that, at low flow rates the effect of rotation is dominant and hence a large vortex is formed, while at high flow rates the phenomenon is reversed.

\section{CONCLUSIONS}

A mathematical model based on steady two-dimensional incompressible Navier-Stokes equations has been used to predict the flow pattern inside a backward-curved blade centrifugal pump. An explicit, finite difference scheme has been developed to solve the Navier-Stokes equations presented in polar coordinate system. The pressure field solution is based on the artificial compressibility approach in which a time derivative pressure term is introduced into the mass conservation equation. A computer code has been developed and used to predict the 
flow pattern inside the pump and to determine the pump characteristics. The experimental investigation showed good agreement with theoretical predictions.

\section{REFERENCES}

1. Wahba W.A., "Flow Pattern in A Centrifugal Pump used in Turbo-Pump Feed System", M.Sc. Thesis, Military Technical College, 1997.

2. Chorin A.J., "A Numerical Method for Solving Incompressible Viscous Flow Problems", J. Comp. Phys., Vol. 2, pp. 12-26, 1967.

3. Hirsch C., and Warzee G., "A Finite-Element Method for Through Flow Calculations in Turbomachines", Transactions of the ASME, Journal of Fluids Engineering, pp. 403-410, 1976.

4. Lakshminarayana B., "An Assessment of Computational Fluid Dynamic Techniques in the Analysis and Design of Turbomachinery - The 1990 Freeman Scholar Lecture", Transactions of the ASME, Journal of Fluids Engineering, Vol. 113, pp. 315-352, 1991.

5. Lotfy, A.H. "Pressure Fluctuations in Radial Flow Centrifugal Pumps at Part Loads", M.Sc. Thesis, Military Technical College, 1981.

6. Anderson D.A., Tannehill J.C., and Pletcher R.H., Computational Fluid Mechanics and Heat Transfer, McGraw Hill Book Company, Second Edition, 1984.

7. Abdalla H., "A Theoretical and Experimental Investigation of the Regenerative Pump with Aerofoil Blades", Ph.D. Thesis, The Royal Military College of Science, 1981.

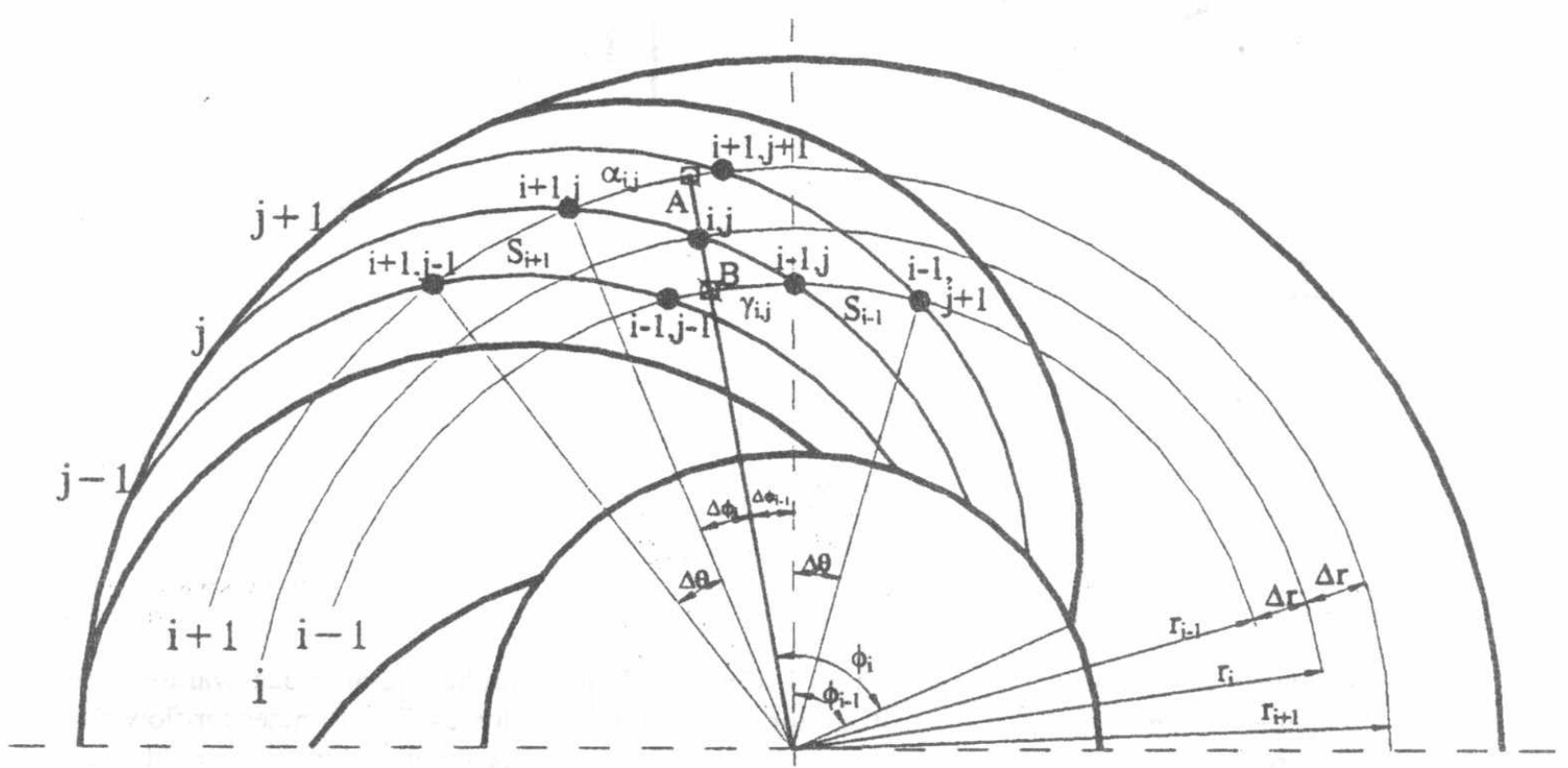

Fig. 1 Definition of the computational grid. 


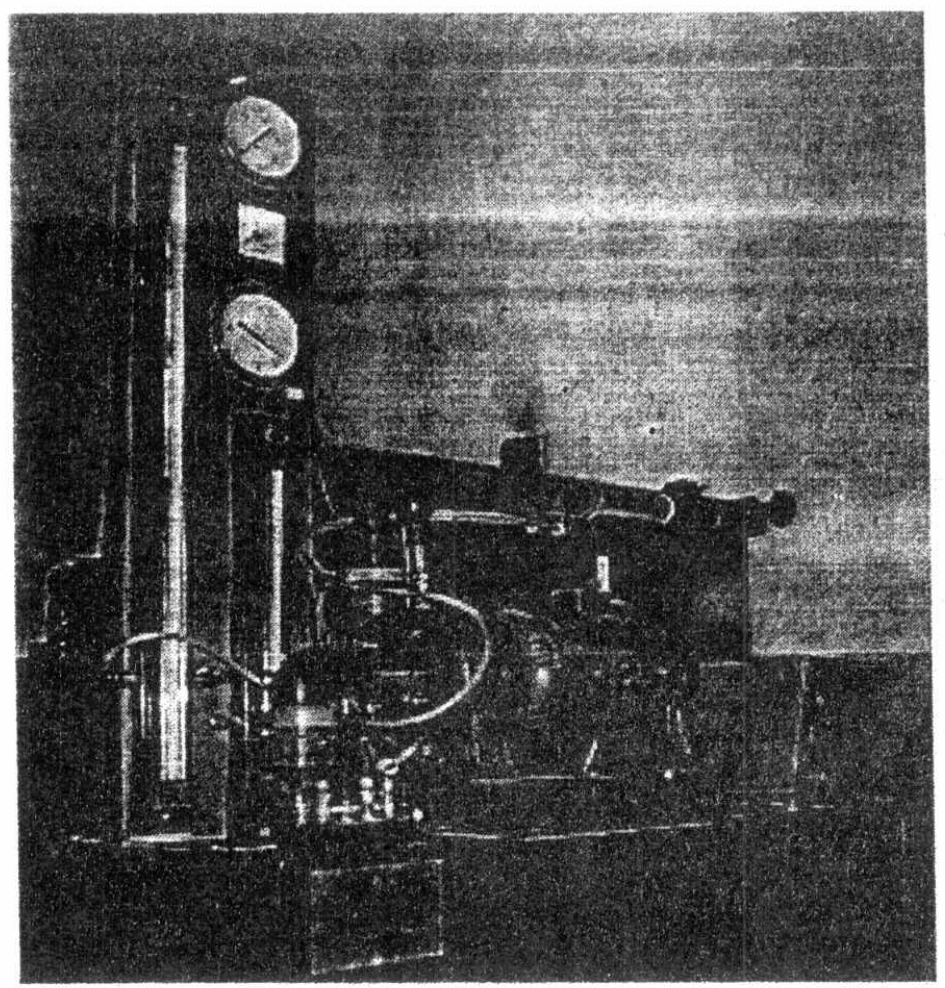

Fig. 2 Test rig photograph.

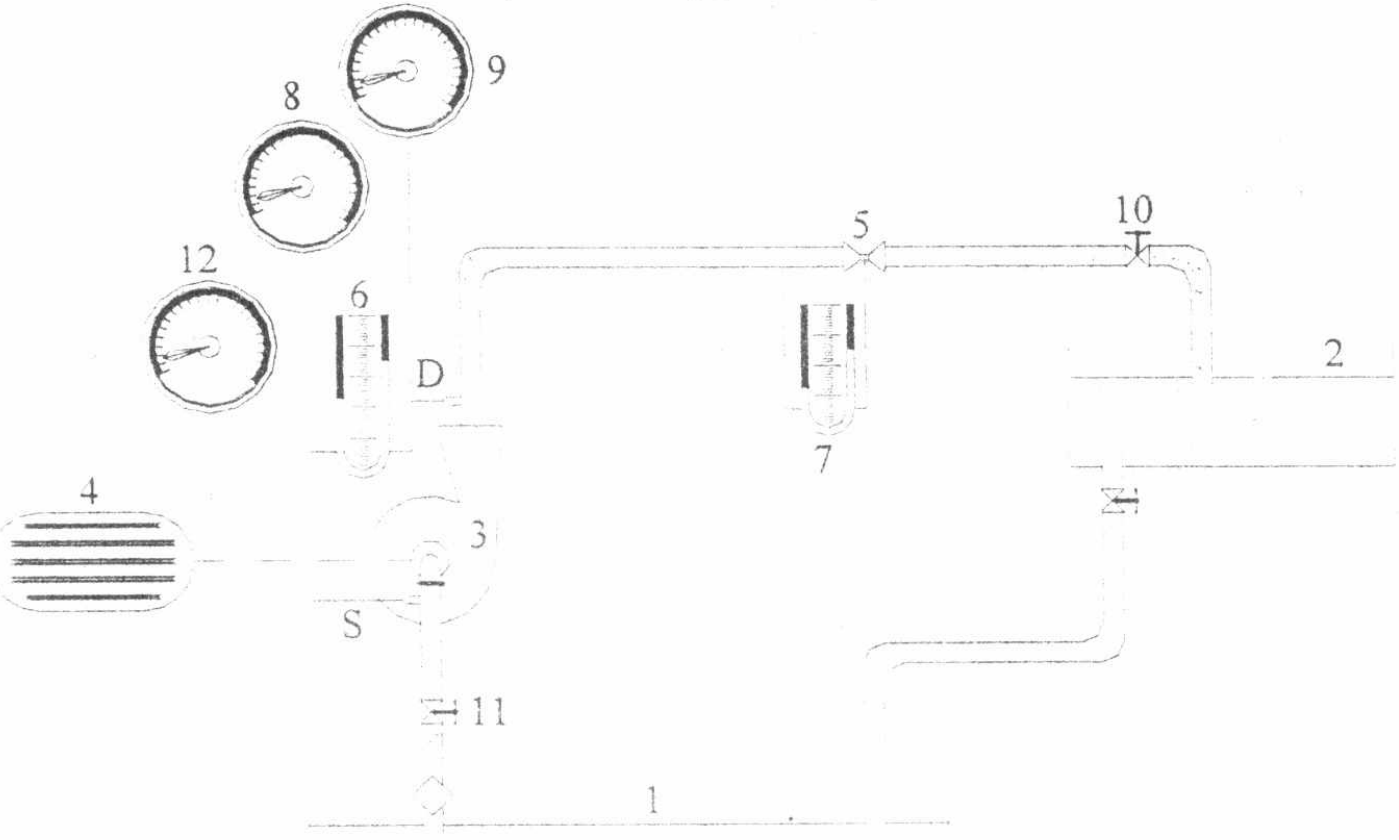

1- Sump tank; 2-Volumetric measuring tank; 3-Centrifugal pump (either radial or backward-curved blade impeller pump can be used); 4- DC motor with speed controller; 5-Venturimeter for flow rate and velocity measurement; 6- One-meter $U$ tube mercury manometer for differential head measurement; 7- A half-meter $U$ tube mercury manometer installed with the venturimeter; 8- Suction pressure gauge $\left(-5.0\right.$ to $\left.0.0 \quad \mathrm{mH}_{2} \mathrm{O}\right)$; 9- Delivery pressure gauge $\left(0.0\right.$ to $\left.20.0 \mathrm{mH}_{2} \mathrm{O}\right) ; 10$ - Delivery control valve; 11 - Suction valve; 12 -Electric tachometer ( 0 to 3000 r.p.m*).

Fig. 3 Test rig schematic diagram. 


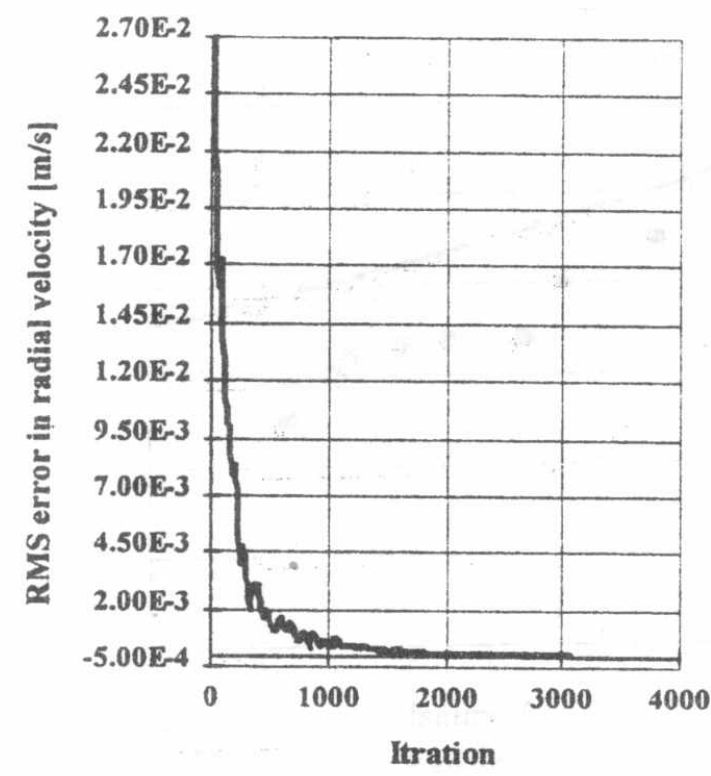

Fig. 4a Convergence of RMS error in radial velocity component.

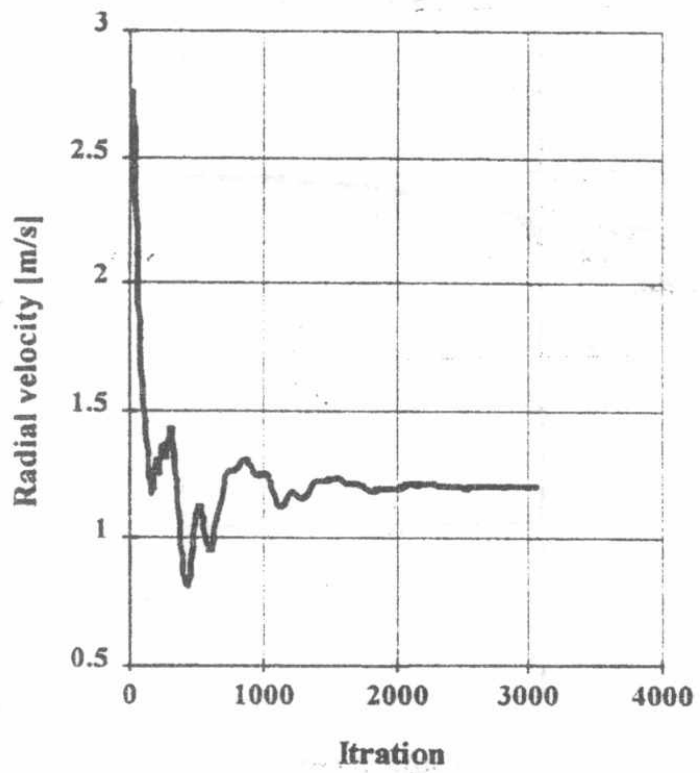

Fig. $4 c$ Convergence of the radial velocity component near leading edge.

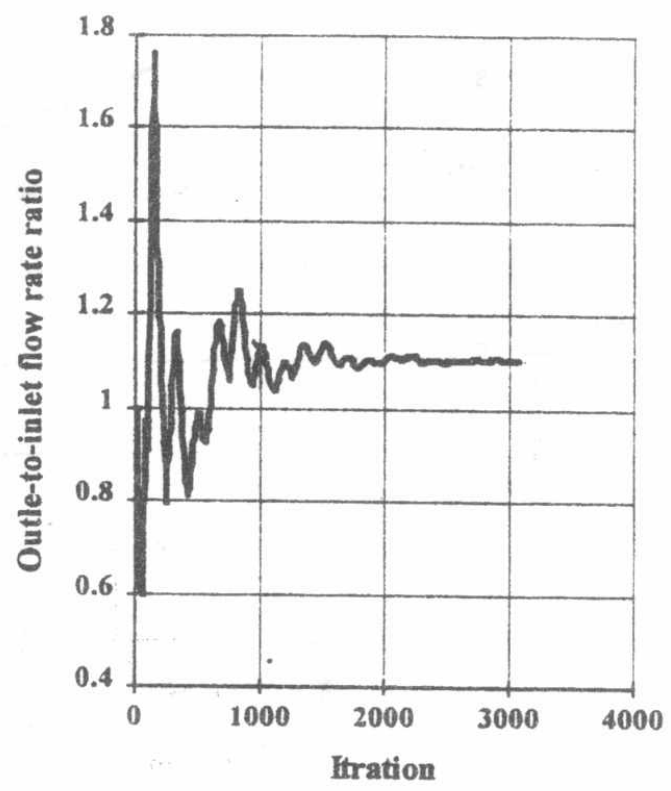

Fig. 4 b Convergence of outlet-to-inlet flow rate ratio.

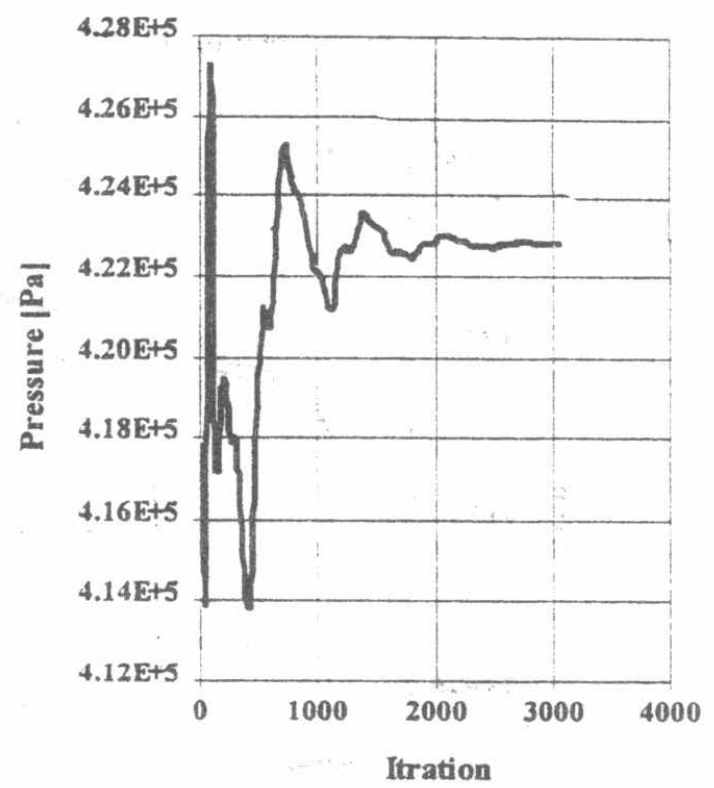

Fig. 4d Convergence of the static pressure at upstream boundary. 


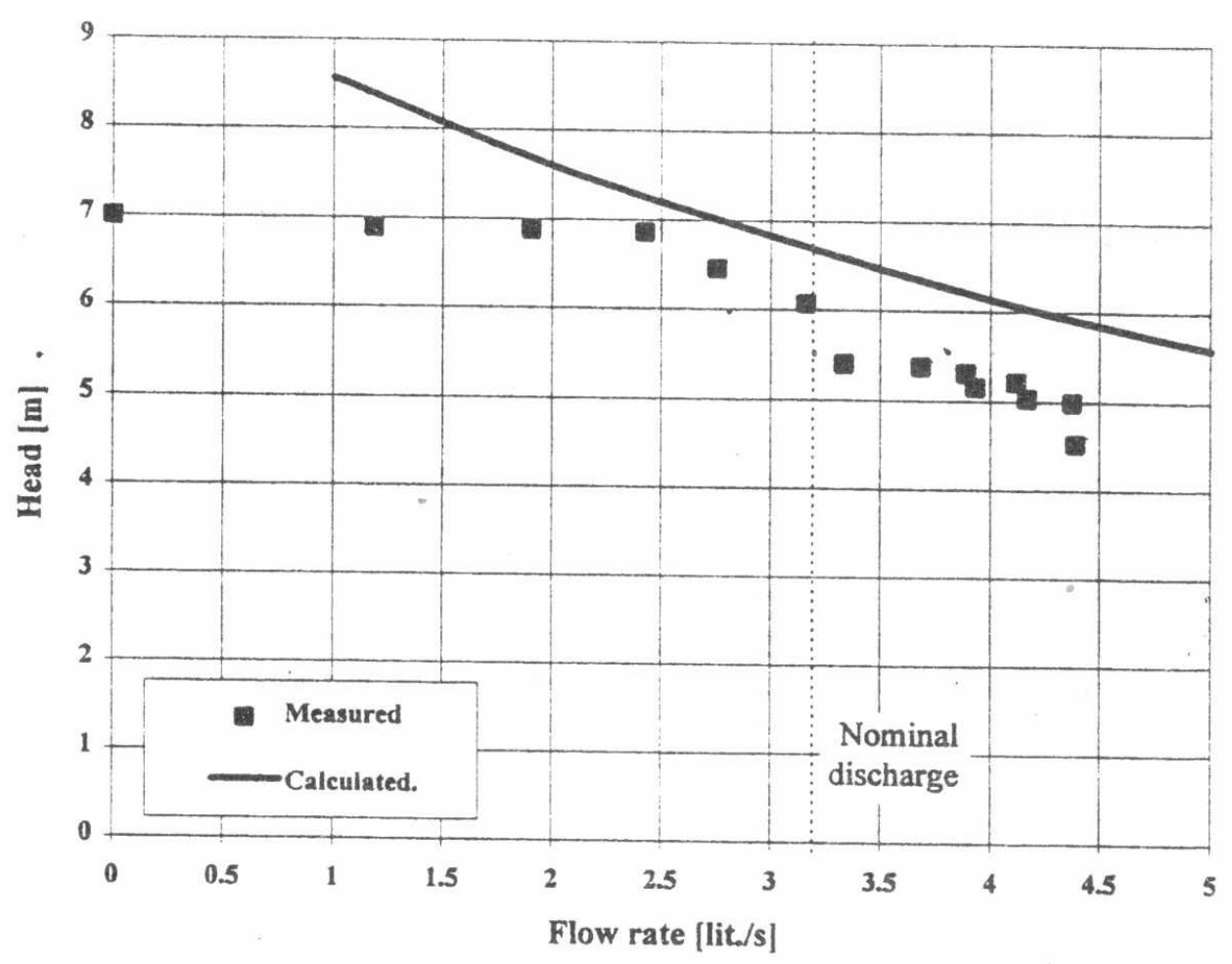

Fig. 5 Comparison between the predicted pump performance and the measured values.

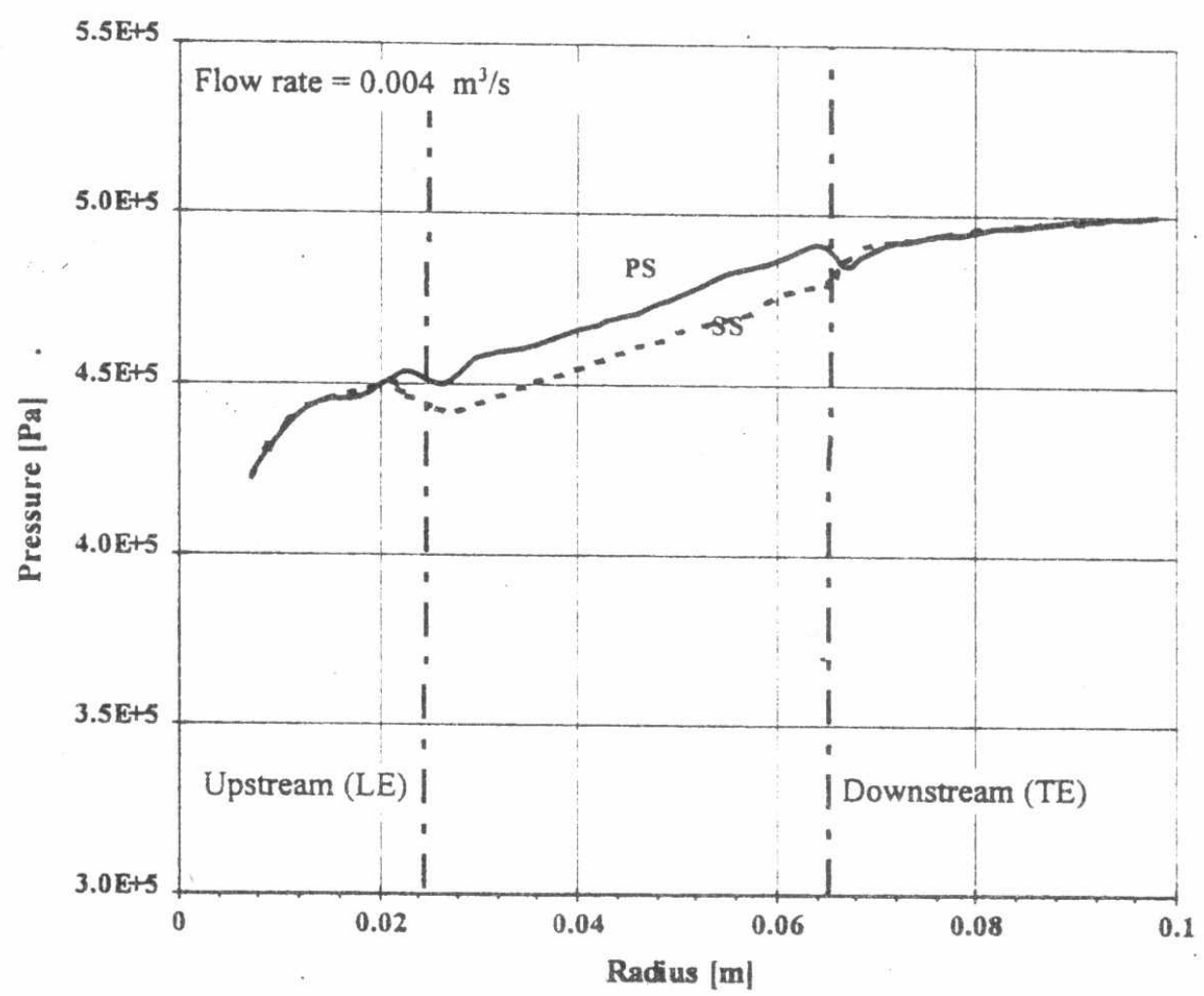

Fig. 6 The static pressure distribution on the pressure and suction sides of the impeller. 

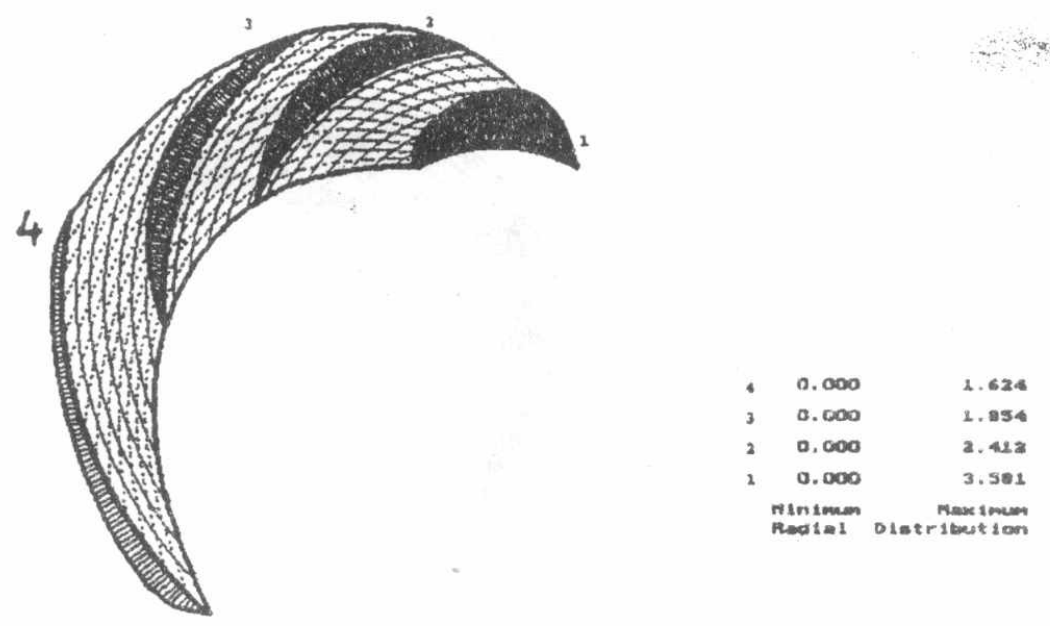

a. Radial relative velocity
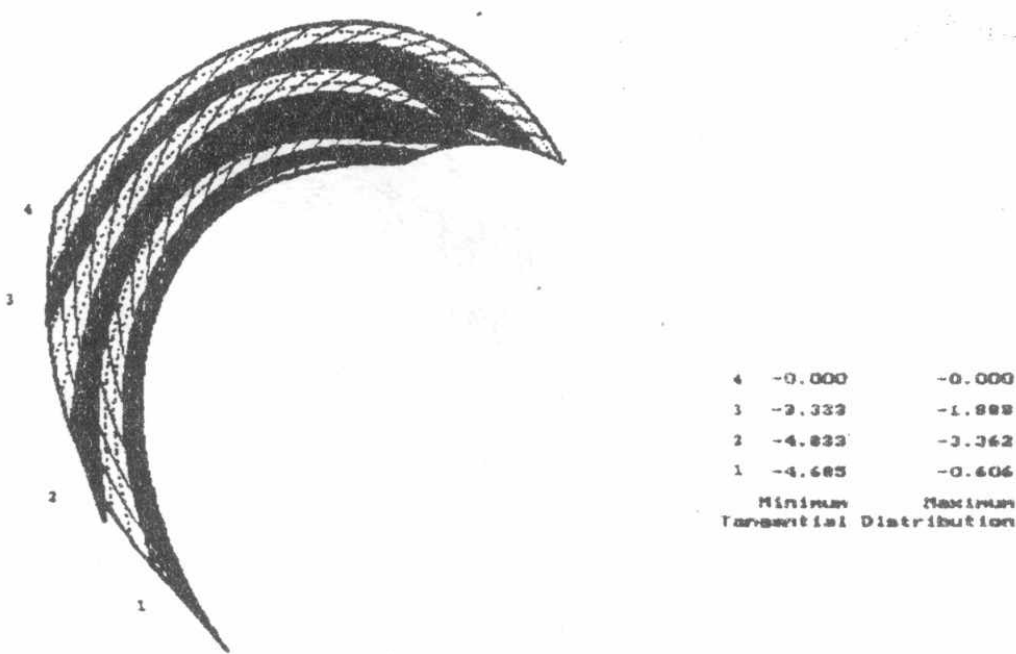

b. Tangential relative velocity.
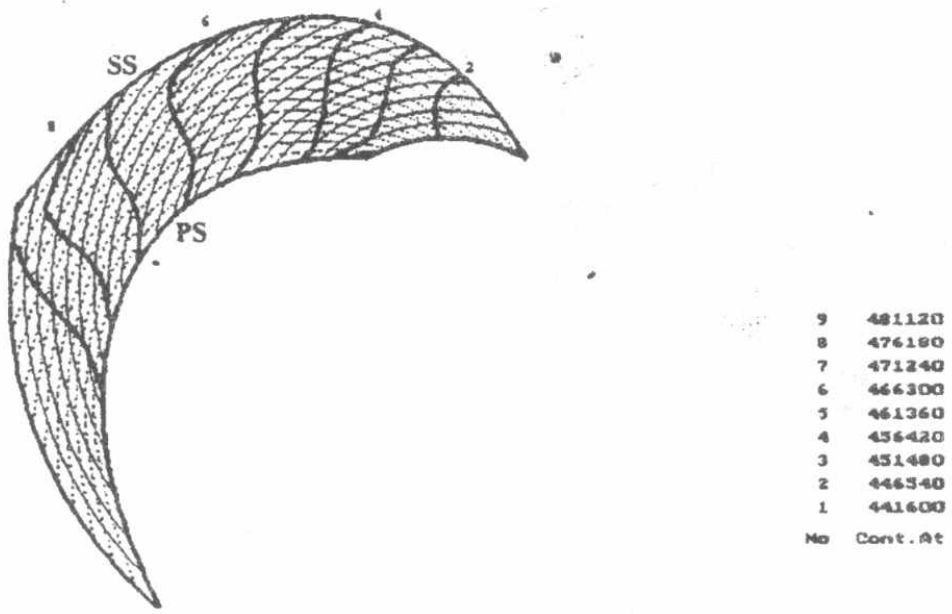

c. Pressure cont ours.

Fig. 7 Distributions of relative velocities and pressure inside impeller blade passage $\left(\right.$ flow rate $=0.004 \mathrm{~m}^{3} / \mathrm{s}$ ) 


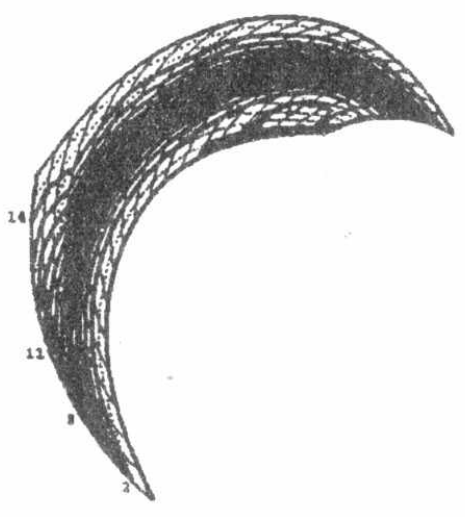

Flow rate $=0.001 \mathrm{~m}^{3} / \mathrm{s}$.

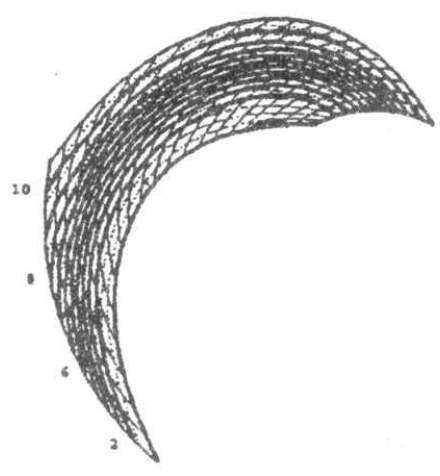

Flow rate $=0.003 \mathrm{~m}^{3} / \mathrm{s}$.
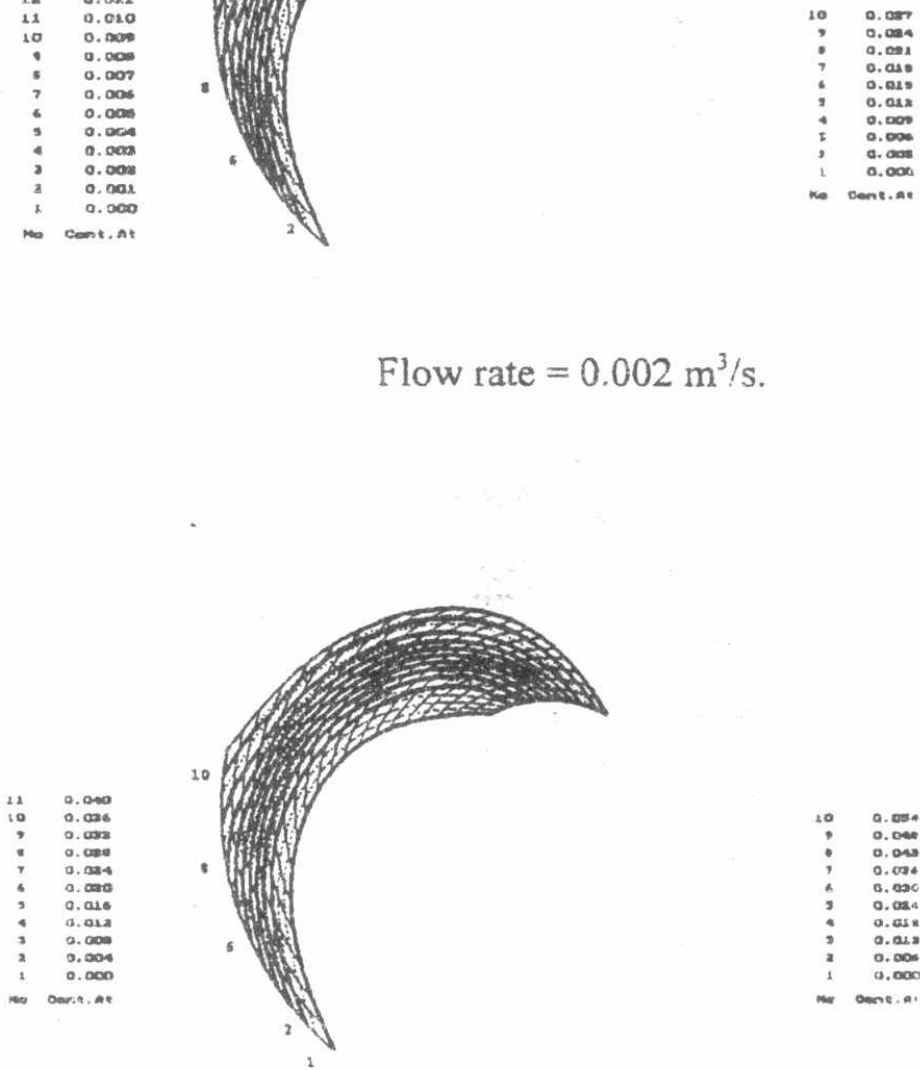

Flow rate $=0.002 \mathrm{~m}^{3} / \mathrm{s}$

Flow rate $=0.004 \mathrm{~m}^{3} / \mathrm{s}$
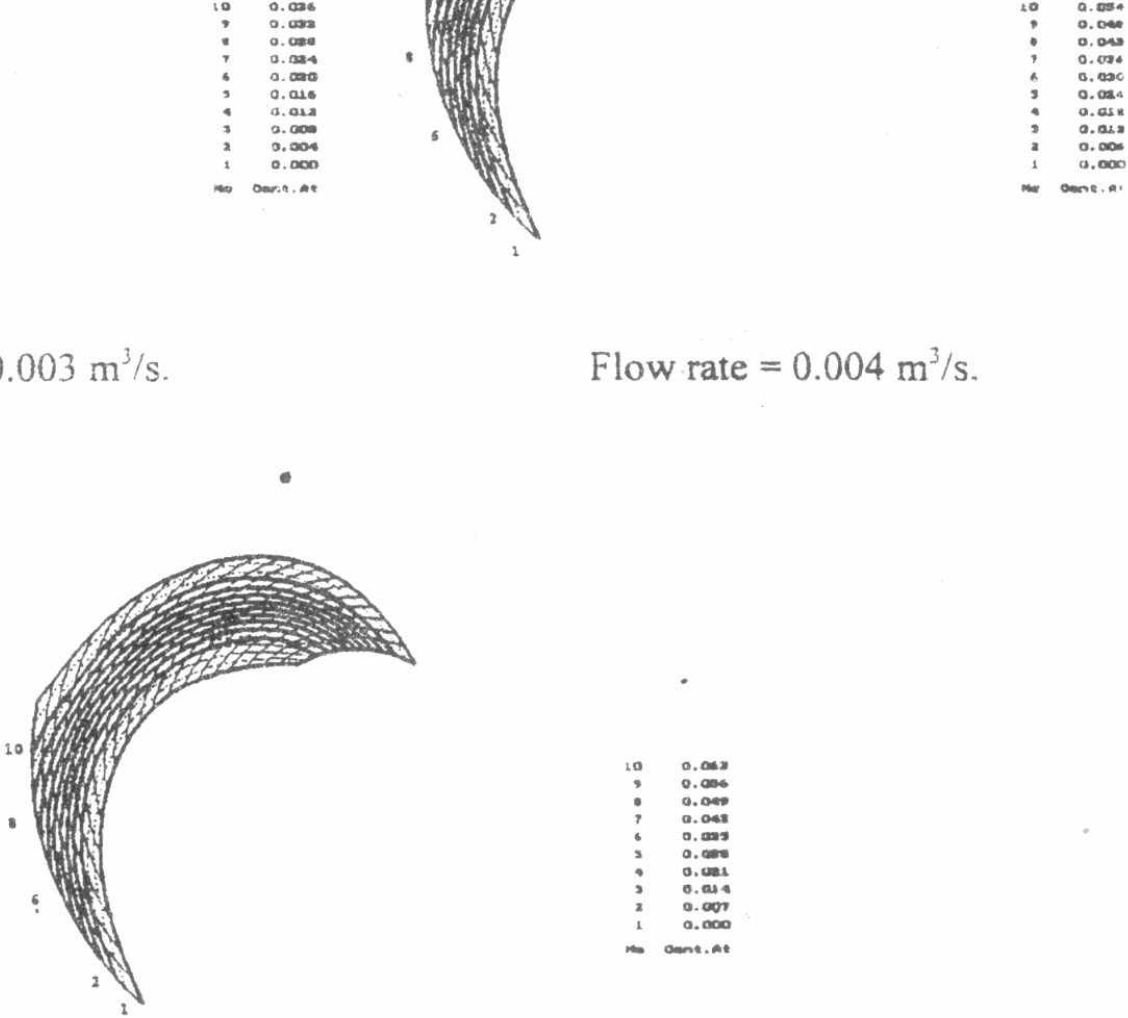

Flow rate $=0.005 \mathrm{~m}^{3} / \mathrm{s}$.

Fig. 8 Flow patteren at different flow rates 